\title{
Intraoperative Cone Beam CT in Hybrid Operating Room Set-Up as An Alternative to Postoperative CT for Pedicle Screw Breach Detection
}

\author{
Simon Peh ${ }^{1}$, Julian Pfarr ${ }^{2}$, Mathis Wegner ${ }^{1 *}$, Jost Philipp Schäfer ${ }^{2}$, Jan-Hendrik Christensen ${ }^{1}$, \\ Anindita Chatterjae ${ }^{3}$, Rami Nachabe ${ }^{3}$, Andreas Seekamp ${ }^{1}$ and Sebastian Lippross ${ }^{1}$ \\ ${ }^{1}$ Department of Orthopedics and Trauma Surgery, University Hospital Schleswig-Holstein, Arnold-Heller-Strasse 3, 24105 Kiel, \\ Germany \\ ${ }^{2}$ Department of Radiology and Neuroradiology, University Hospital Schleswig-Holstein, Arnold-Heller-Strasse 3, 24105 Kiel, Germany \\ ${ }^{3}$ Image Guided Therapy Systems, Philips Healthcare, Veenpluis 4-6, 5684 PC, Best, The Netherlands \\ *Corresponding author: Mathis Wegner, Department of Orthopedics and Trauma Surgery, University Hospital Schleswig-Holstein, \\ Arnold-Heller-Strasse 3,24105 Kiel, Germany.
}

\section{ARTICLE INFO}

Received: 慧 July 21, 2021

Published: 幽 July 28, 2021

Citation: Simon Peh, Julian Pfarr, Mathis Wegner, Jost Philipp Schäfer, Jan-Hendrik Christensen, et al., Intraoperative Cone Beam CT in Hybrid Operating Room SetUp as An Alternative to Postoperative CT for Pedicle Screw Breach Detection. Biomed J Sci \& Tech Res 37(4)-2021. BJSTR. MS.ID.006022.

Abbreviations: CBCT: Cone-Beam CT; ED: Effective Dose; RDSR: Dose Structured Report; ROC: Receiver Operating Characteristic; CI: Confidence Intervals.

\begin{abstract}
Background: CT is considered the gold standard for detecting pedicle breach. However, CBCT may be a viable and low radiation dose alternative, to provide intraoperative feedback to surgeons to permit in-room revisions of misplaced screws.

Methods: To assess the ability and reliability of intraoperative cone-beam CT (CBCT) from a robotic $\mathrm{C}$-arm in a hybrid operating room (OR) two hundred forty-one pedicle screws were inserted in cervical, thoracic and lumbar spine of 7 cadavers, followed by CBCT and CT imaging. The CT images served as the standard of reference. Agreement on screw placement between both imaging systems was assessed using Cohen's Kappa coefficient ( $\kappa)$. Sensitivity, specificity, receiver operating characteristic (ROC), area under the empirical and fitted ROC curves (AUC) were computed to assess CBCT as a diagnostic tool compared to CT. The patient effective radiation dose (ED) was calculated for comparison. A systematic literature review was performed to provide perspective to the obtained results.
\end{abstract}

Results: Almost perfect agreement in assessing pedicle screw grading between CBCT and CT was observed ( $\kappa=0.84)$. The sensitivity and specificity of CBCT were 0.84 and 0.98 , respectively. The AUC derived from the empirical and fitted ROC curves were 0.95 and 0.96 , respectively.

Conclusion: Intraoperative CBCT by C-arm in a hybrid OR is highly reliable in identification of screw placement at significant dose reduction.

Keywords: Hybrid Operating Room; Cone Beam Computed Tomography; Pedicle Screw Placement; Pedicle Breach; Minimally Invasive Spine Surgery; Dose Reduction.

\section{Introduction}

Accurately positioned pedicle screws offer optimal biomechanical fixation, making them a well-accepted treatment choice for a variety of spine pathologies [1,2]. However, up to $42 \%$ of pedicle screws were reported to be malpositioned $[3,4]$.
While initially silent clinically, these may eventually present with an unstable construct, reduced fusion, pseudoarthrosis and accelerated adjacent-level degeneration [5]. In comparison to CT, radiography detects only $52 \%$ of misplaced screws [6,7], making intraoperative CT imaging necessary, especially for complex 
deformity, to avoid revision surgeries. Availability of intraoperative 3D imaging resulted in revision of $9 \%$ of screws intraoperatively, corresponding to $35 \%$ of the treated patients. Lower threshold for intraoperative revisions based on objective intraoperatively available imaging data, leads to fewer secondary revision surgeries [8]. Avoidance of postoperative revisions makes the initial investment in intraoperative 3D imaging technologies economically more attractive [9].

Hybrid operating rooms (OR) equipped with a motorized C-arm coupled with a radiolucent surgical table as well as with integrated navigation capabilities, have been recently used for spine surgery [10]. The C-arm provides intraoperative cone-beam CT (СВCT) imaging. The purpose of this cadaver study was to determine the diagnostic performance of intraoperative CBCT using a C-arm in a hybrid OR for identifying screw misplacements in comparison to gold-standard postoperative CT. To put our results in perspective, a sytematic literature review of studies comparing intraoperative 3D imaging for screw misplacements with postoperative CT was conducted.

\section{Materials and Methods}

\section{Surgical Technique}

Surgeries were performed by one surgeon in a hybrid OR (AlluraClarity Flexmove, Philips, the Netherlands), (Figure 1). Cadavers were placed prone on the operating table, adhesive skin markers applied and a planning CBCT scan obtained. Pedicle screw trajectories were planned using Augmented Reality Surgical Navigation system (ARSN, Philips, the Netherlands) [11]. Pedicles were instrumented bilaterally from C7 to L5 using a tracked Jamshidi needles, aligned along the planned trajectory. Cannulated pedicle screw sizes were $5.5 \times 40 \mathrm{~mm}$ from $\mathrm{C} 7$ to T9; and $6.5 \mathrm{x}$ $45 \mathrm{~mm}$ from T10 to L5.

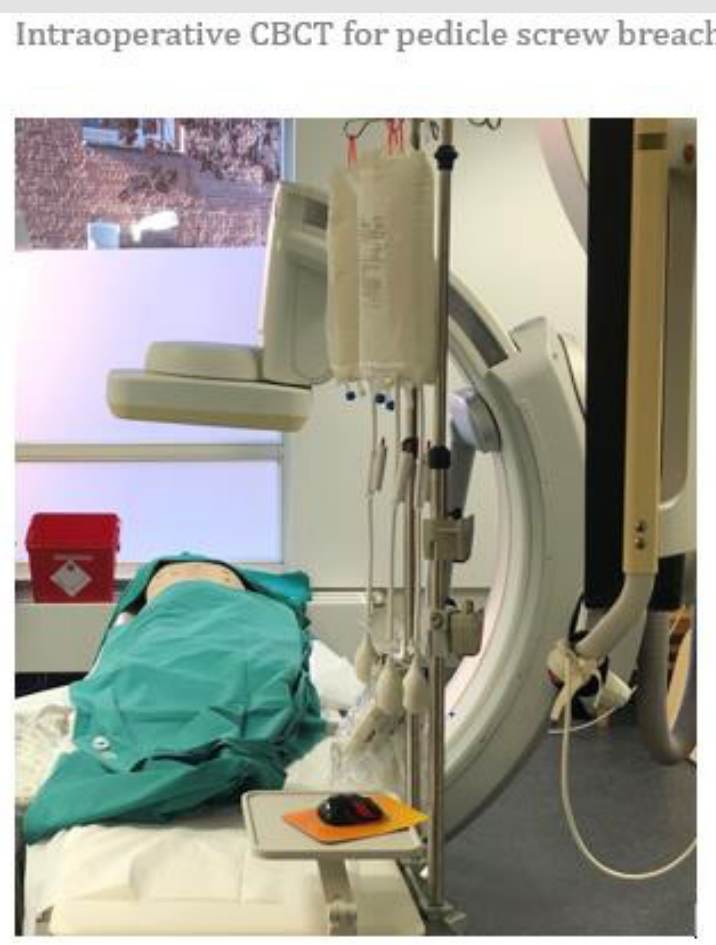

Figure 1: Set up of the hybrid OR and the patient positioning on the table.

\section{CBCT Imaging: The Study Group}

The $\mathrm{C}$-arm was positioned on the side, orthogonal to the operating table along the axial plane of the patient and rolled in this plane to perform CBCT optimized for spine imaging (XperCT Spine, Philips, the Netherlands). The CBCT reconstruction is the result of 302 x-ray projections acquired over a 180-degree clockwise rotation, starting with the $\mathrm{x}$-ray tube on the right side of the table. The acquisition settings were 120 nominal $\mathrm{kV}$ tube voltage and modulated tube current-exposure time (mAs) generated by the automatic exposure control which is estimated based on patient size thickness at each angular projection during the rotation. Radiation dose-area products were collected from the ra-diation Dose Structured Report (RDSR) and converted to patient Effective Dose (ED). The CBCT reconstruction corresponds to a $512 \times 512 \times 396$ matrix with isotropic voxels of $0.49 \mathrm{~mm}$. Consequently, the dimension of the imaged region of interest (ROI) corresponds to a $25 \times 25 \mathrm{~cm}$ in the axial plane by $19.5 \mathrm{~cm}$ in the cranial-caudal direction [12], (Figure 2). 
Intraoperative $\mathrm{CBCT}$ for pedicle screw breach detection

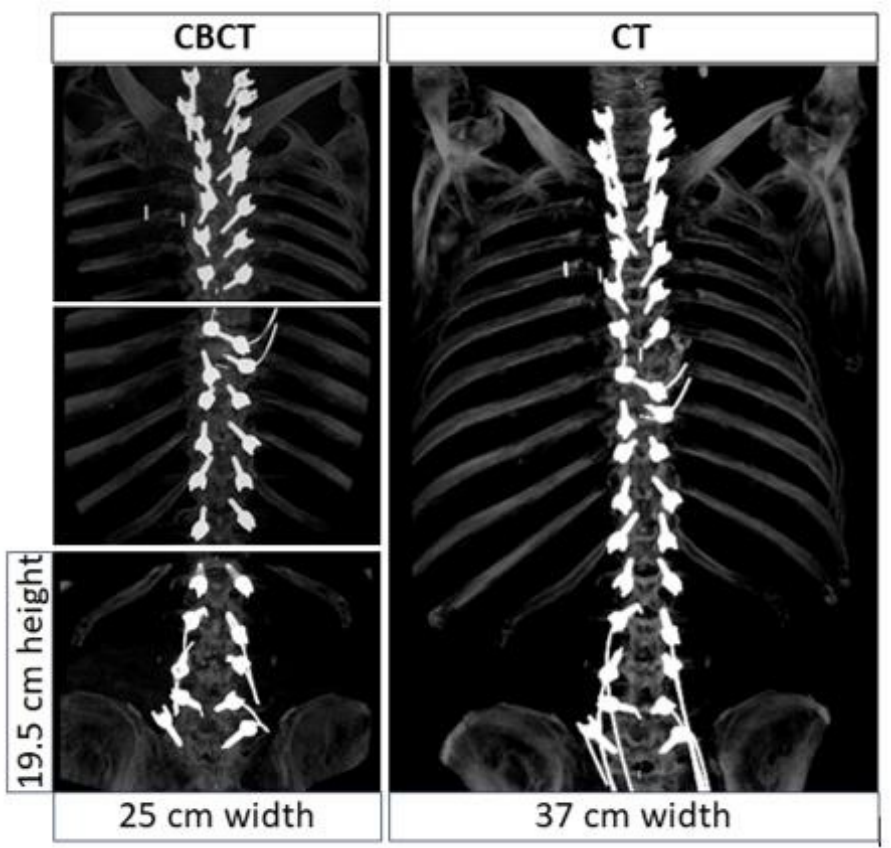

Figure 2: Imaging of the whole spine of one cadaver from the CBCT and CT.

\section{CT: The Reference Group}

Default settings for spine imaging on a 16-slice CT scanner was used to image the cadavers (Brillance 16, Philips, the Netherlands). CT image reconstructions were made based on a helical acquisition at $120 \mathrm{kV}$ tube voltage acquisition and a modulated tube currentexposure time (mAs), derived from the anterio-posterior and lateral scout scans. Radiation dose-length products were collected from the RDSRs and converted to patient ED. The CT image reconstructions were performed at $0.75 \mathrm{~mm}$ slice thickness and di-mension of the reconstructed ROI image was $37 \times 37 \mathrm{~cm}$ in the axial plane, in craniacaudal length direction covering the whole instrumented spine, (Figure 2).

\section{Data Analysis}

The CBCT and CT images were reviewed on a PACS system with 3D volumes displayed in a multi-planar format. The window width and level were set to optimize visualization. Screw position assessments were performed using the Gertzbein grading [13]:

- $\quad$ grade 0 (screw within the pedicle without cortical breach)

- $\quad$ grade 1 (0-2 mm breach, minor perforation including cortical encroachment)

- $\quad$ grade 2 (2-4 $\mathrm{mm}$ breach, moderate breach)

- $\quad$ grade 3 (more than $4 \mathrm{~mm}$ breach, severe misplacement).

Each screw was assigned a grade from 0 to 3, for each imaging modality. Consequently, a $4 \times 4$ contingency table to compare the similarity in rating was created. (Figure 3) illustrates an example of each of the 4 grades on both CBCT and CT from the diagonal of the contingency table (i.e. agreement between both imaging modality for each grade). All images in (Figure 3) were displayed for comparison at equal display settings optimized for bone and hardware imaging. Grades 0 and 1 were considered clinically accurate, whereas grades 2 and 3 were considered misplaced. 


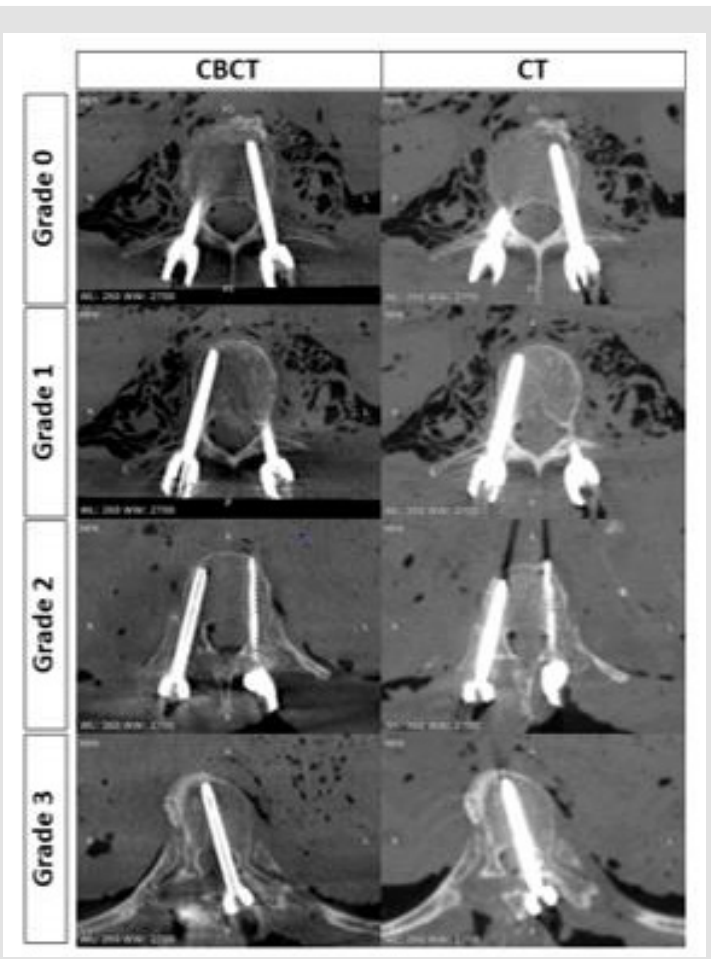

Figure 3: Images displaying all the 4 grades from $\mathrm{CBCT}$ and $\mathrm{CT}$ for the same screw. The chosen screws for each grade have agreement between both imaging modalities.

\section{Statistical Analysis}

Cohen's Kappa coefficient ( $\mathrm{K}$ ) was calculated to assess the agreement between CBCT and CT based on the contingency tables and was classified according to Landis et al. [14] as detailed in (Table 1). Sensitivity was defined as the proportion of misplaced screws detected on CBCT which were confirmed as such on CT; whereas specificity was defined as the proportion of screws accurately placed according to CBCT which was confirmed as such on CT.
Receiver operating characteristic (ROC) analysis was performed and area under the empirical and fitted ROC curves (AUC) were computed to assess performance of CBCT as a diagnostic tool whose performance was classified according to Hanley et al. [15] (Table 1). Two-sided Fisher's exact and Wilcoxon signed rank tests were used for comparison where appropriate. Two-sided AUC comparison test was performed. Confidence Intervals (CI) were computed at 95\% and expressed between brackets. A p-value (p) less than 0.05 was considered as significant.

Table 1: Relation between Cohen's Kappa coefficient and strength of intermodality agreement as well as relation between area under the receiver operating characteristic curve value and strength of diagnosis.

\begin{tabular}{|c|c|c|c|}
\hline Cohen's Kappa coefficient & $\begin{array}{c}\text { Strength of agreement } \\
\text { [Ref Landis 1997] }\end{array}$ & $\begin{array}{c}\text { Area under the curve (AUC) } \\
\text { [Ref Hanley 1982] }\end{array}$ & Perfect \\
\hline 1 & Perfect & $=1.0$ & Excellent \\
\hline $0.8-1.0$ & Almost perfect & $0.9-1.0$ & Good \\
\hline $0.6-0.8$ & Substantial & $0.8-0.9$ & Fair \\
\hline $0.4-0.6$ & Moderate & $0.7-0.8$ & Poor \\
\hline $0.2-0.4$ & Fair & $0.6-0.7$ & Fail \\
\hline$<0.0$ & Slight & $0.5-0.6$ & $<0.5$ \\
\hline
\end{tabular}

\section{Literature Review}

A systematic review was conducted in order to perform a proper comparison with all the existing literature on assessing the diagnostic performance of intraoperative 3D imaging versus
CT. The review was performed using PubMed Central, PubMed, Embase, Web of Science, and Cochrane with the following search strategy: ("pedicle screw placement" AND "sensitivity" AND "specificity" AND "imaging"). After filtering for duplicates from the various search engines, 5 exclusion criteria were applied 
during abstract screening: (a) articles not written in English, (b) conference abstracts, (c) articles that do not report about pedicle screw (e.g. sacroiliac screws), (d) articles that are not comparative studies, and (e) studies that do not consider an intraoperative 3D system as study group (e.g. 2D radiography, electromyography, etc.). After text screening, papers that did not report clinical accuracy for screw placement by using a Gertzbein-like grading i.e. (2-mm increment scale relative to breach) were excluded as also data with inconsistency or missing in-formation. All preclinical and clinical studies were included. Literature data with available contingency tables, or with detailed numbers of screws per grade for each group from which contingency tables could be generated, were used to calculate sensitivity, specificity, and AUC and statistical tests performed to ensure a fair diagnostic performance comparison between all studies.

\section{Results}

Two hundred forty-one cannulated pedicle screws were placed percutaneously: 3 (1\%), 168 (70\%), and 70 (29\%) in the cervical, thoracic, and lumbar spine respectively.

\section{Accuracy Comparison Between CBCT and CT}

Table 2: Contingency table comparing pedicle screw placement assessment between CBCT and CT.

\begin{tabular}{|l|c|c|c|c|c|}
\hline \multicolumn{2}{|c|}{} & \multicolumn{4}{c|}{ CT } \\
\cline { 3 - 6 } & Grade 0 & Grade 1 & Grade 2 & Grade 3 \\
\hline & Grade 1 & 134 & 13 & 2 & 0 \\
\hline CBCT & Grade 2 & 24 & 22 & 3 & 2 \\
\hline & Grade 3 & 1 & 3 & 5 & 12 \\
\hline & Grade 4 & 0 & 0 & 2 & 17 \\
\hline
\end{tabular}

All cadavers had their full instrumented spine imaged with 3 CBCT as seen in comparison with CT, (Figure 2). Table 2 corresponds to the contingency table comparing the grading assessment between both imaging modalities for each screw. Out of the 241 placed screws, $134,22,6$, and 17 screws were rated by both imaging modalities as grade $0,1,2$, and 3 , respectively: yielding a total of 179 screws with equal ratings. With respect to screws considered as accurate placement on CT, twenty-four screws and one screw were rated as grade 1 and 2 respectively on CBCT while they were rated grade 0 on CT, whereas thirteen screws and three screws were rated as grade 0 and 2 respectively on CBCT while they were rated as grade 1 on CT. With respect to screws considered as misplaced on CT, two screws were rated grade 0 and three screws were graded 1 on CBCT while they were rated as grade 2 on CT. Finally, two and twelve screws were rated as grade 1 and 2 on CBCT while they were rated as grade 3 on CT. The Cohen's Kappa coefficient was $\kappa=0.84$ [0.75-0.93] indicating almost perfect agreement in assessing accuracy between CBCT and CT.
The agreement in the lumbar spine was almost perfect $(\mathrm{k}=0.87$ $[0.70-1.00])$ as well as in the thoracic spine ( $\mathrm{k}=0.83$ [0.59-0.82]). The sensitivity and specificity of СВCT were 0.84 [0.70-0.93] and 0.98 [0.95-0.99], respectively. Sensitivity and specificity in the lumbar spine (0.89 [0.52-1.00] and 0.98 [0.91-1.00]) were higher than in the thoracic spine (0.83 [0.66-0.93] and 0.98 [0.94-1.00]) but not statistically different ( $\mathrm{p}=1$ for both). The AUC derived from the empirical and fitted ROC curves were 0.95 [0.91-1.00] and 0.96 [0.92-1.00], respectively; indicating CBCT to be an excellent diagnostic tool for pedicle screw placement compared to CT.

\section{Radiation Dose of the Subjects}

The patient ED in CBCT imaging was significantly lower than CT for all 7 cadavers (median $8.2 \mathrm{mSv}$ vs $12.9 \mathrm{mSv}, \mathrm{p}<0.05)$ ). The average ED was 7.2 $\pm 5.4 \mathrm{mSv}$ in CBCT vs $14.3 \pm 3.4 \mathrm{mSv}$ in CT with a $53 \%$ ED reduction on $\mathrm{CBCT}$.

\section{Literature Review and Comparison}

The initial database search identified 117 studies without any duplicates. 104 articles were excluded after scrutiny of abstracts. The two most common reasons for exclusion were articles not being comparative studies $(n=35)$ and the study group not corresponding to an intraoperative 3D imaging such as 2D radiography, EMG, or other techniques $(n=35)$. Fifteen studies were not related to pedicle screws (e.g. iliac screws), and an equal amount corresponded to conference abstracts. Four abstracts corresponded to papers not written in English. From the remaining 13 articles, 5 other exclusions were applied at text screening level. The most common reason of exclusion was not using a Gertzbein-like grading with 2 mm increment for breach $(n=5)$ [16-20]. One study was excluded because of inconsistency between the data in the abstract and in the text [21]. One study did not use a grading scale but revision surgery as surrogate parameter for screw misplacement [22].

Another study did not confirm whether the obtained accuracy was indeed breach $<2 \mathrm{~mm}$ or all breaches (i.e. grades 1, 2 and 3) [23]. One study did not provide enough information to compute accuracy for breach $<2 \mathrm{~mm}$ and considered all breaches as misplaced screws [24]. Finally, there were 4 studies that were discussed in this review [25-28], (Figure 4). Two cadaveric studies compared intraoperative CBCT vs macroscopic dissection $[25,26]$. In one study results were compared to CT as well [26]. Two clinical studies compared CBCT vs CT $[27,28]$. Details are presented in Table 3, showing sensitivity, specificity, and AUC where applicable. Sensitivity and specificity in literature ranged between 0.69-0.86 and between $0.71-1.00$, respectively. The AUC could only be derived from one study [28] with an available contingency table yielding AUC from the empirical and fitted ROC curves of 0.91 [0.83-0.98] and 0.93 [0.87-1.00] respectively. Figure 5 shows a comparison of the ROC curves and corresponding AUC. 
Table 3: Literature data comparison. C, T, and L correspond to cervical, thoracic, and lumbar spinal levels, respectively. K corresponds to Cohen's Kappa coefficient. AUCe and AUCf correspond to the area under the empirical and fitted receiver operating characteristic curve, respectively. NA indicates not available data.

\begin{tabular}{|c|c|c|c|c|c|c|c|}
\hline Reference & Comparison & $\begin{array}{c}\text { Number of screws } \\
(\mathrm{C} / \mathrm{T} / \mathrm{L})^{1}\end{array}$ & Sensitivity & Specitivity & $\mathrm{K}[\mathrm{CI}]$ & AUCe [CI] & AUCf [CI] \\
\hline Santos et al. (2012) & $\begin{array}{l}\text { CBCT vs } \\
\text { macroscopic } \\
\text { dissection }\end{array}$ & $\begin{array}{c}416 \\
\text { (NA/NA/NA) }\end{array}$ & $\begin{array}{l}0.69 \\
\text { (NA) }\end{array}$ & $\begin{array}{l}0.71 \\
\text { (NA) }\end{array}$ & NA & NA & NA \\
\hline Sarwahi et al. (2017) & $\begin{array}{l}\text { CBCTvs } \\
\text { macroscopic } \\
\text { dissection }\end{array}$ & $\begin{array}{c}288 \\
(0 / N A / N A)\end{array}$ & $\begin{array}{l}0.77 \\
\text { (NA) }\end{array}$ & $\begin{array}{l}0.84 \\
(\mathrm{NA})\end{array}$ & NA & NA & NA \\
\hline Sarwahi et al. (2017) & СВCT vs CT & $\begin{array}{c}288 \\
(0 / N A / N A)\end{array}$ & $\begin{array}{l}0.86 \\
\text { (NA) }\end{array}$ & $\begin{array}{l}0.91 \\
\text { (NA) }\end{array}$ & NA & NA & NA \\
\hline $\begin{array}{c}\text { Fujimori et al. } \\
\text { (2017) }\end{array}$ & СВCT vs CT & $\begin{array}{c}203 \\
(0 / 79 / 124)\end{array}$ & $\begin{array}{c}0.7 \\
{[0.35-0.92]}\end{array}$ & $\begin{array}{c}0.95 \\
{[0.90-0.97]}\end{array}$ & $\begin{array}{l}0.54 \\
(\mathrm{NA})\end{array}$ & NA & NA \\
\hline $\begin{array}{l}\text { 2017-Cordemans } \\
\text { et al.2 }\end{array}$ & CBCT vs CT & $\begin{array}{c}348 \\
(0 / 135 / 213) \\
\end{array}$ & $\begin{array}{c}0.76 \\
{[0.57-0.90]}\end{array}$ & $\begin{array}{c}1 \\
{[0.98-1.00]}\end{array}$ & $\begin{array}{c}0.83 \\
{[0.72-0.95]}\end{array}$ & $\begin{array}{c}0.91 \\
{[0.83-0.98]}\end{array}$ & $\begin{array}{c}0.93 \\
{[0.87-1.00]}\end{array}$ \\
\hline This study & CBCT vs CT & $\begin{array}{c}241 \\
(3 / 168 / 70)\end{array}$ & $\begin{array}{c}0.84 \\
{[0.70-0.93]}\end{array}$ & $\begin{array}{c}0.98 \\
{[0.95-0.99]}\end{array}$ & $\begin{array}{c}0.84 \\
{[0.75-0.93]}\end{array}$ & $\begin{array}{c}0.95 \\
{[0.91-1.00]}\end{array}$ & $\begin{array}{c}0.96 \\
{[0.92-1.00]}\end{array}$ \\
\hline
\end{tabular}

Note: CI: confidence interval; K: Kappa

CBCT: cone beam computed tomography; CT computed tomography.

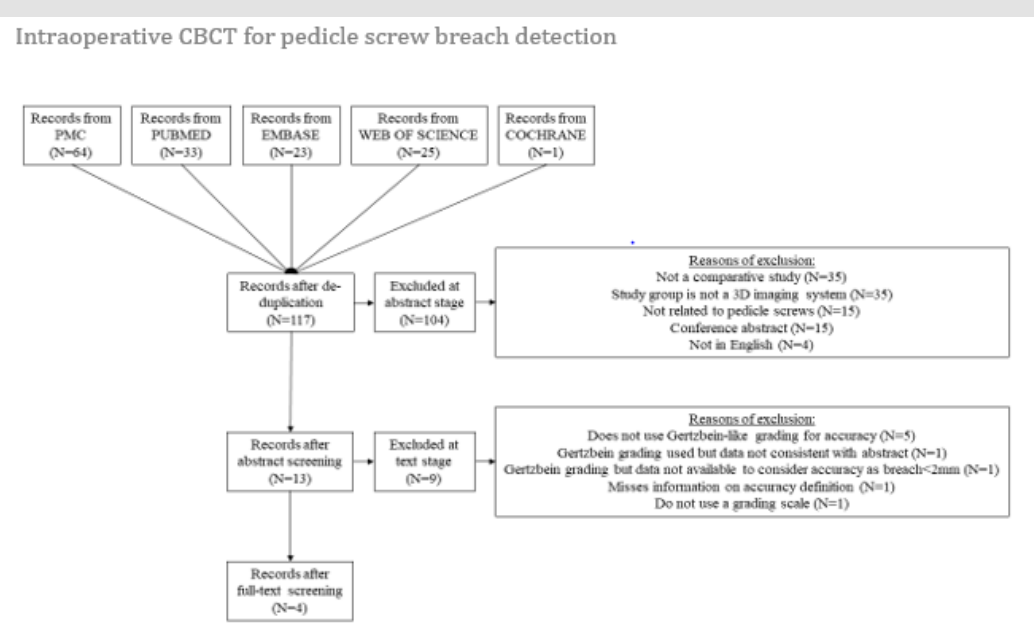

Figure 4: Strategy and inclusion/exclusion criteria for the literature search review.

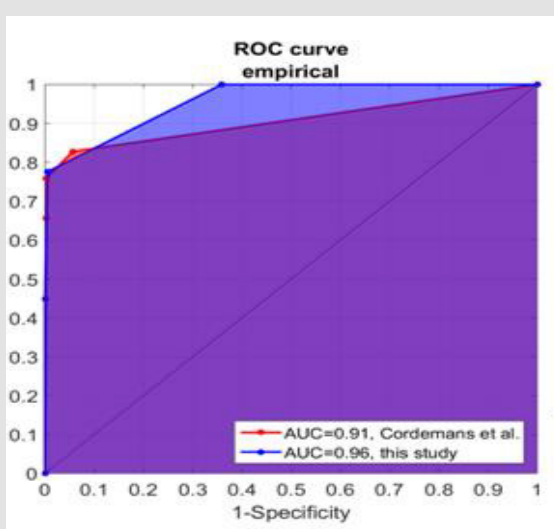

a.)

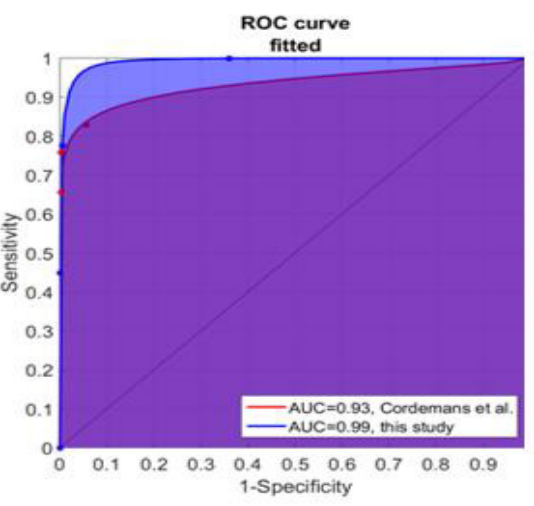

b)

Figure 5: Comparison of the ROC curves and the corresponding AUC. (a) Comparison of empirical ROC curves of Cordemans et. al. and this study; (b) Comparison of fitted ROC curves of Cordemans et. al. and this study. 


\section{Discussion}

Hybrid ORs support multidisciplinary use of 2D and 3D imaging and navigation for open and minimal invasive procedures [10]. This cadaver study sought to assess the diagnostic performance of CBCT from a C-arm within a hybrid OR compared to diagnostic CT. Unlike mobile C-arm with CBCT capability and mobile CT, the C-arm system in the hybrid OR has an image acquisition and processing chain comparable to diagnostic CT scanners, enabling better management of image quality and radiation dose exposure [29]. While macroscopic dissection is the gold reference for evaluating pedicle screw placement, CT is the clinically viable alternative [26]. Choosing a cadaver study over a clinical study allowed for immediate comparison between CBCT and CT. Furthermore, cadaver studies allow uniformity of CT and CBCT protocols on the same scanner to ensure fair comparisons. In clinical studies, follow-up scans are often performed several months after surgery. Bone remodeling and regrowth or screw loosening occurs during the follow-up period which can influence the Gertzbein grading introducing thus possibilities for comparison errors.

The almost perfect inter-modality agreement of this study was suggestive of CBCT as a viable replacement for postoperative CT. The sensitivity obtained for the CBCT images was 0.84 , indicating that $84 \%$ of misplaced screws detected on CT, were detected on CBCT. Of the 7 screws that were missed as a misplaced screw in CBCT, 6 were in the thoracic region. A specificity of 0.98 , indicates in $2 \%$ of the cases, CBCT identified screws as misplaced when they are assessed to be well placed on CT. The screws that had the degree of cortical wall penetration overestimated in the CBCT, were mainly in the thoracic region. Interestingly, 5 of 7 screws were missed as misplaced on CBCT and 3 of 4 instances where the screw was labelled as misplaced on CBCT but a well-placed screw on CT, had a difference of 1 grade. In Gertzbein, there is an increment of $2 \mathrm{~mm}$ in the amount of dis-placement between the consecutive grades. It is likely that the metal artefacts and the small pedicle sizes in the thoracic region, could potentially be the cause for the $2 \mathrm{~mm}$ error in the assessment. A higher sensitivity compared to specificity suggests that CBCT based assessment tends to underestimate pedicle breaches compared to CT. Clinically, it is important to detect a truly misplaced screw.

Therefore, sensitivity is the most important parameter. However, sensitivity and specificity should both be compared together to get the overall picture. AUC analysis from ROC curves is the radiologically accepted technique to assess a system's diagnostic performance described by a single number, as it considers sensitivity-specificity for all grades. The CBCT used in this study yielded AUCs suggestive of its excellence as a diagnostic tool for assessing screw placement during surgery. The conducted systematic literature review yielded 4 studies meeting the search criteria. The sensitivity and specificity of this study was in the upper range from the reported values in the literature, (Table 3). The Cohen's Kappa and the AUC are indicators of the strength of agreement and diagnosis comparisons respectively. The strength of agreement was superior to the study performed with a mobile CBCT by Fujimori et al. [27] and comparable to a study performed with CBCT in a hybrid OR [28]. The study by Cordemans et al. is the only study from which the AUC could be derived for comparison of diagnostic strength [28].

These values were lower than in this current study but not statistically significant ( $\mathrm{p}=0.20$ and $\mathrm{p}=0.27$ for the empirical and fitted AUC, respectively). The fitted AUC accounts for the continuous nature of measuring breach while the em-pirical AUC only takes the $2 \mathrm{~mm}$ increment as defined by Gertzbein. Inclusion of a high number of misplaced screws $(18 \%$ with a grade 2 or higher breach as per Gertzbein) and screws in the cervical or upper thoracic regions where the pedicle sizes are small (36\% screws were in these regions) provide a better evaluation of the technology. This is reflected by the higher confidence interval compared to literature, thereby providing a higher reliability of sensitivity. The CBCT offers significant patient ED reduction (53\% reduction) compared to CT. In institutions where a threshold for patient radiation dose exposure exists for perioperative procedures, one can justify the use of intraoperative CBCT as a diagnostic tool at a lower radiation dose exposure compared to CT, without altering diagnostic judgement of screw placement.

Cervical screws were limited in this study and detection of pedicle breach in the cervical region may be more challenging, given the smaller pedicle diameter and the shoulder superimposition in the image. Additional studies addressing other factors impacting image quality such as contrast-to-noise ratio, severity of metal artefacts, image reconstruction algorithm, and radiation dose protocol should be performed to complete the diagnostic evaluation of CBCT versus CT.

\section{Conclusions}

Intraoperative CBCT by $\mathrm{C}$-arm in a hybrid OR is highly reliable in identification of screw placement at significant dose reduction. A systematic literature search demonstrated superior performance compared to the existing CBCT of mobile C-arm systems confirming reported performance by another study using a hybrid OR system.

\section{Author Contributions}

SP Study setup, data acquisition, data analysis, writing. JP Study plan-ning, data acquisition, data analysis. MW manuscript writing, submission. JPS Study planning, data acquisition, data analysis. JHC data analysis, statistics, drafting manuscript. AC Study set-up, data acquisition, data analysis. RN Study setup, data acquisition, data analysis. AS Study planning, manuscript writing. SL Study setup, data acquisition, data analysis, writing. All authors have read and agreed to the published version of the manuscript. 


\section{Funding}

Traveling expenses for the study for Simon Peh and Sebastian Lippross were paid by Philips Healthcare. Rami Nachabe and Anindita Chatterjea are employed by Philips Healthcare, which has a vested interest in the product described in this manuscript. The research has been performed as a part of a research agreement between University Hospital Schleswig-Holstein, Campus Kiel, Germany and Philips Healthcare. For the remaining authors, none were declared beyond this research agreement of their employer.

\section{Institutional Review Board Statement}

The study was conducted according to the guidelines of the Declaration of Helsinki. Ethics approval was obtained for a cadaver study from the ethics committee of the Christian-AlbrechtUniversity Kiel, Germany.

\section{Informed Consent Statement}

Informed consent was obtained from all subjects involved in the study before decease.

\section{Data Availability Statement}

Not applicable

\section{Conflicts of Interest}

The authors declare no conflict of interest. The funders had no role in the design of the study; in the collection, analyses, or interpretation of data; in the writing of the manuscript, or in the decision to publish the results.

\section{References}

1. Gaines RW (2000) The use of pedicle-screw internal fixation for the operative treatment of spinal disorders. J Bone Joint Surg Am 82(10): $1458-1476$

2. Inceoğlu S, Montgomery WH, Clair S (2011) Pedicle screw insertion angle and pullout strength: comparison of two proposed strategies. J Neurosurg Spine 14(5): 670-676.

3. Upendra BN, Meena D, Chowdhury B (2008) Outcome-based classification for assessment of thoracic pedicular screw placement. Spine 33(4): 384-390.

4. Gelalis ID, Paschos NK, Pakos EE, Avraam Ploumis, Theodoros A Xenakis, et al. (2012) Accuracy of pedicle screw placement: a systematic review of prospective in vivo studies comparing free hand, fluoroscopy guidance and navigation techniques. Eur Spine J 21(2): 247-255.

5. Açikbas SC, Arslan FY, Tuncer MR (2003) The effect of transpedicular screw misplacement on late spinal stability. Acta Neurochir 145(11): 949-955.

6. Sarwahi V, Ayan S, Amaral T (2017) Can postoperative radiographs accurately identify screw misplacement? Spine Deform 5(2): 109-116.

7. Faber GL, Place HM, Mazur RA (1995) Accuracy of pedicle screw placement in lumbar fusions by plain radiographs and computed tomography. Spine. 20(13): 1494-1499.
8. Bydon M, Xu R, Amin AG (2014) Safety and efficacy of pedicle screw placement using intraoperative computed to-mography: consecutive series of 1148 pedicle screws. J Neurosurg Spine 21(3): 320-328.

9. Dea N, Fisher CG, Batke J, Marcel F S Dvorak, John T Street, et al. (2016) Economic evaluation comparing intraoperative cone beam CT-based navigation and conventional fluoroscopy for the placement of spinal pedicle screws: a patient-level data cost-effectiveness analysis. Spine J 16(1): 23-31.

10. Berazaluce A, Hanke R, Von Allmen D (2019) The State of the Hybrid Operating Room: Technological Acceleration at the Pinnacle of Collaboration Pediatric surgery. Curr Surg Rep. 7: 7.

11. Peh S, Chatterjea A, Pfarr J, Andreas Seekamp, Sebastian Lippross, et al. (2020) Accuracy of augmented reality surgical navigation for minimally invasive pedicle screw insertion in the thoracic and lumbar spine with a new tracking device. Spine J 20(4): 629-637.

12. Nachabe R, Strauss, K, Schueler B (2019) Radiation Dose and Image Quality Comparison During Spine Surgery with Two Different, Intraoperative 3D Imaging Navigation Systems. J Appl Clin Med Phys 20(2): 136-145.

13. Gertzbein SD, Robbins SE (1990) Accuracy of pedicular screw placement in vivo. Spine 15(1): 11-14.

14. Landis J, Heyman E, Koch G (1978) Average Partial Association in ThreeWay Contingency Tables: A Review and Discussion of Alternative Tests. International Statistical Review 46(3): 237-254.

15. Hanley JA, McNeil, BJ (1982) The meaning and use of the area under a receiver operating characteristic (ROC) curve. Radiology 143(1): 29-36.

16. Nevzati E, Fandino J, Schatlo B, Luca Remonda, Ali-Reza Fathi, et al. (2017) Validation and accuracy of intraoperative CT scan using the Philips AlluraXper FD20 angiography suite for assessment of spinal instrumentation. Br J Neurosurg 31(6): 741-746.

17. Beck M, Mittlmeier T, Gierer P, Harms C, Gradl, G. (2018) Benefit and accuracy of intraoperative 3D-imaging after pedicle screw placement: a prospective study in stabilizing thoracolumbar fractures. Eur Spine J 18(10): 1469-77.

18. Beck M, Rotter R, Gradl G, Markus Kröber, Thomas Mittlmeier, et al. (2012) Reliability and consequences of intraoperative 3D imaging to control positions of tho-racic pedicle screws. Arch Orthop Trauma Surg 132(10): 1371-1377.

19. Yoo J, Ghanayem A, Petersilge C, Lewin J (1997) Accuracy of using computed tomography to identify pedicle screw placement in cadaveric human lumbar spine. Spine 22(22): 2668-2671.

20. Kim HS, Heller JG, Hudgins PA (2003) The Accuracy of Computed Tomography in Assessing Cervical Pedicle Screw Placement. Spine 28(21): 2441-2446.

21. Garber S, Bisson E, Schmidt M (2012) Comparison of Three-Dimensional Fluoroscopy versus Postoperative Computed To-mography for the Assessment of Accurate Screw Placement after Instrumented Spine Surgery. Global Spine J 2(2): 95-98.

22. Salama A, Amin, M, Soliman A (2019) Postoperative 320 multi-slice computed tomography in assessment of pedicle screw insertion in thoraco-lumbar fixation. Egyptian Journal of Radiology and Nuclear Medicine 50: 1 .

23. Rao G, Brodke DS, Rondina M (2002) Comparison of computerized tomography and direct visualization in thoracic pedicle screw placement. J Neurosurg 97: 223-226.

24. Wang M, Kim A, Liu C (2004) Reliability of three-dimensional fluoroscopy for detecting pedicle screw violations in the thoracic and lumbar spine. Neurosurgery 54(5):1138-1142. 
25. Santos E, Ledonio C, Castro C (2012) The Accuracy of Intraoperative 0-arm Images for the Assessment of Pedicle Screw Postion. Spine 37(2): E119-E125.

26. Sarwahi V, Payares M, Wendolowski S, Yungtai Lo, Terry D Amaral, et al. (2017) Low-Dose Radiation 3D Intraoperative Imaging: How Low Can We Go? An O-Arm, CT scan. Cadaveric Study. Spine 42(22): E1311-E1317.

\section{ISSN: 2574-1241}

DOI: $10.26717 /$ BJSTR.2021.37.006022

Mathis Wegner. Biomed J Sci \& Tech Res

(c) (P) This work is licensed under Creative

Submission Link: https://biomedres.us/submit-manuscript.php
27. Cordemans V, Kaminski L, Banse X (2017) Accuracy of a new intraoperative cone beam CT imaging technique (Artis zeego II) compared to postoperative CT scan for assessment of pedicle screws placement and breaches detection. Eur Spine J 26(11): 2906-2916.

28. Jaju PP (2015) Cone Beam Computed Tomography: A clinician's Guide to 3D Imaging. Jaypee Brothers pp. 34-40.0d modis ant illa conseque sundunto berum cus, nonestrum as magnimi llibus.

$\begin{array}{ll}\text { BIOMEDICAL } & \text { Assets of Publishing with us } \\ \text { RESEARCHES } & \text { - Global archiving of articles } \\ \text { - Immediate, unrestricted online access }\end{array}$

